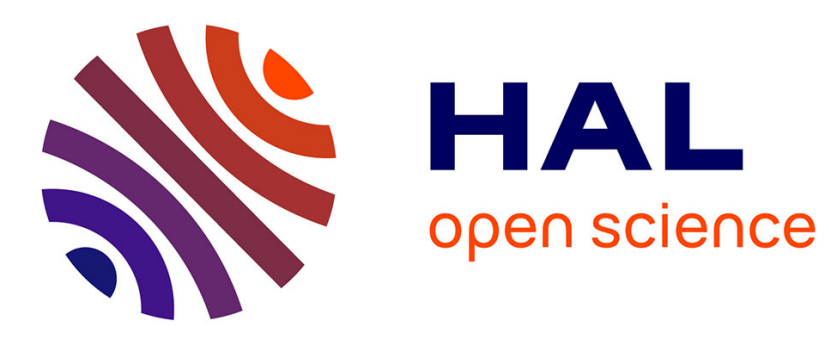

\title{
Propelled strings: rising from friction
}

\author{
Nicolas Taberlet, Jérémy Ferrand, Nicolas Plihon
}

\section{To cite this version:}

Nicolas Taberlet, Jérémy Ferrand, Nicolas Plihon. Propelled strings: rising from friction. Physical Review Letters, 2019, 123 (144501), 10.1103/PhysRevLett.123.144501 . hal-02398889

\section{HAL Id: hal-02398889 https://hal.science/hal-02398889}

Submitted on 8 Dec 2019

HAL is a multi-disciplinary open access archive for the deposit and dissemination of scientific research documents, whether they are published or not. The documents may come from teaching and research institutions in France or abroad, or from public or private research centers.
L'archive ouverte pluridisciplinaire HAL, est destinée au dépôt et à la diffusion de documents scientifiques de niveau recherche, publiés ou non, émanant des établissements d'enseignement et de recherche français ou étrangers, des laboratoires publics ou privés. 


\title{
Propelled Strings: Rising from Friction
}

\author{
Nicolas Taberlet, Jérémy Ferrand, and Nicolas Plihon $\odot$ \\ Univ Lyon, ENS de Lyon, Univ Claude Bernard Lyon 1, CNRS, Laboratoire de Physique, F-69342 Lyon, France
}

(Received 18 April 2019; published 4 October 2019)

\begin{abstract}
The shape of closed strings and chains propelled at a constant velocity and launched at an angle relative to gravity is studied experimentally, theoretically, and numerically. At low velocity, strings adopt a shape close to the well-known catenary, while at high velocity, they can rise to a nearly horizontal profile. We show that the latter regime can be counterintuitively attributed to aerodynamic effects, although the ambient air exerts no lift on a string moving longitudinally along its profile. A theoretical approach along with numerical simulations confirms these observations and allows one to predict the shape of any closed string or chain. Moreover, depending of the regime, waves rising from any local perturbation along the string may travel either upstream or downstream and seem to die out at the turning point. We show that these observations can be explained by the tension profile along the string, which strongly depends on the aerodynamic effects relative to the weight, and our theoretical analysis allows us to predict the position of the wave front.
\end{abstract}

DOI: 10.1103/PhysRevLett.123.144501

Introduction.-Despite their apparent simplicity, ropes and chains are well-known to display intriguing phenomena due, among other things, to self-interactions or interactions with solid boundaries and surrounding fluids. Surprisingly, chains pulled from a horizontal plane [1] or out of a container [2-6] can form spectacular self-supporting vertical arches. Besides these striking phenomena, understanding the dynamical weight of falling chains in simple configurations - chains falling from a smooth surface [7-9] or from an initial $U$-folded arrangement [10] -have long attracted much attention [11,12]. In textile manufacturing (a major industrial application), a variety of whirling modes is observed in rotating spindles [13]. Several modern applications rely on the dynamics of long and slender objects dragged in fluid environments, such as sonar sensors towed at the end of a long cable attached to a ship $[14,15]$, or aerial systems towed by aircrafts for payload delivery [16], refueling, or atmospheric research [17]. In these situations, the steady-state profile and the temporal dynamics of the cables depend on the hydrodynamic forces, the tension of the cable, the gravity, and the flexural rigidity $[14,15,18,19]$. In this Letter, we investigate the steady-state shape and the wave propagation of disturbances of closed inextensible strings propelled at a constant velocity at an arbitrary angle with gravity. Elongated structures in the propelling direction may then be observed above a critical velocity. Observations from various experimental setups are presented and compared to theoretical predictions, showing a counterintuitive result: the string lifts when air friction exceeds the weight. The respective influence of the weight and the drag on the overall profile is then analyzed using numerical simulations. Finally, the propagation of disturbances along the string are studied in the two limit cases, respectively, dominated by the weight or by the drag force. Understanding the evolution of the tension of the string along the profile allows us to explain why traveling waves can travel either upstream or downstream and to elucidate why they die out at the turning point.

System description.-The shape of closed strings or chains propelled at a high velocity $v$ at an arbitrary angle with the horizontal $\alpha(0)$ is investigated first. A typical experimental arrangement is shown in Fig. 1: a string (or a chain) is guided between two vertical wheels spinning against one another (see Supplemental Material, Video part I [20]). Further technical details are given in Supplemental Material I [20].

Since the propelling wheels are in the vertical $(x, y)$ plane, the string remains in this plane. Therefore, mathematically, the shape can be described by the local angle $\alpha$ with respect to the horizontal, seen as a function of the curvilinear coordinate $s$, measured from the propelling point $A$ at which the string leaves the wheels, to point $B$ at which it comes in contact with the bottom wheel or with the guiding system as Fig. 1, i.e., from 0 to the length $L$.

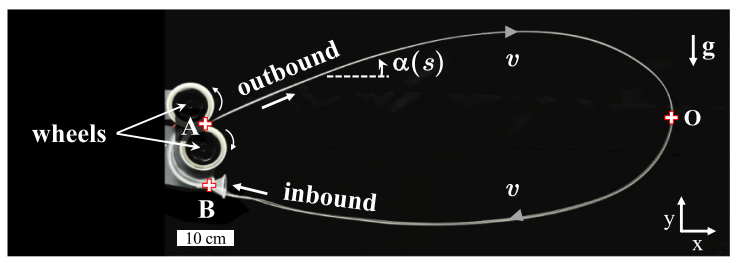

FIG. 1. A light closed $2 \mathrm{~m}$ long string is propelled by two rotating wheels towards the right, at angle $\alpha(0)=27^{\circ}$ relative to the horizontal and velocity $v=9.5 \mathrm{~m} \mathrm{~s}^{-1}$. 

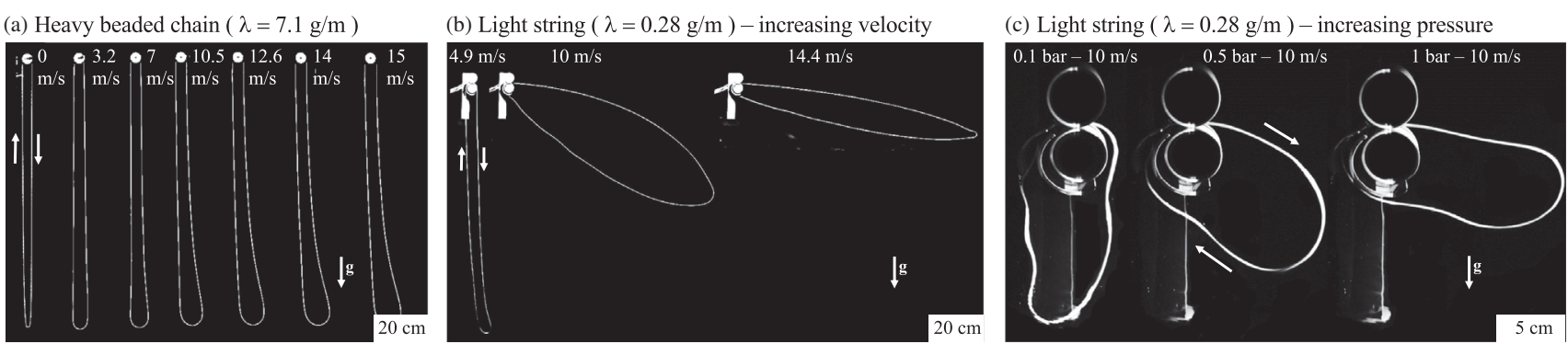

FIG. 2. Steady-state shapes of a $2 \mathrm{~m}$ long (a) heavy beaded chain and (b) light string for increasing velocities. (c) Propelled string (35-cm long) in a vacuum for constant velocity and propelling angle $[\alpha(0)=0]$ for various pressures.

The point at which the string turns around (i.e., for which $\alpha=-\pi / 2$ ), noted $O$, delimits the outbound branch (i.e., the $A O$ section) and the inbound branch $(O B)$. Experimentally, the knot made to close the string may trigger waves in the string (mostly on the inbound branch) as can be seen in the Supplemental Material Video [20] and Fig. 2. For a seamless loop, however, these instabilities disappear.

Governing equations.-The strings and chains used cannot be elongated, and as a consequence, the linear velocity, $v$, remains uniform (although it obviously changes direction) along the length of the string $L$. The flexural rigidity and the internal dissipation of the cotton strings and beaded chains used in our experiment can be neglected (except in the case of high curvature, see Supplemental Material [20] and Fig. 3). Two external forces act on the string: (i) its own weight per unit length $\lambda g-\lambda$ being the linear mass - and (ii) air drag (along the flow) per unit length $\boldsymbol{f}_{d}$. Let us emphasize, again, that, due to the symmetry of a string moving along its tangent, the aerodynamical effects reduce to a drag force without any lift. Introducing the tension $T(s)$ within the string, the equations of motion read (projected on the horizontal, $x$, and vertical, $y$, coordinates) $[15,21]$

$$
\begin{aligned}
d(T \cos \alpha) & =-\lambda v^{2} \sin \alpha d \alpha+f_{d} \cos \alpha d s, \\
d(T \sin \alpha)-\lambda g d s & =\lambda v^{2} \cos \alpha d \alpha+f_{d} \sin \alpha d s .
\end{aligned}
$$

Introducing an effective tension $T_{\text {eff }}=T-\lambda v^{2}$, and a kinetic tension $T_{k}=\lambda v^{2}$, these equations can be rewritten in dimensionless form as
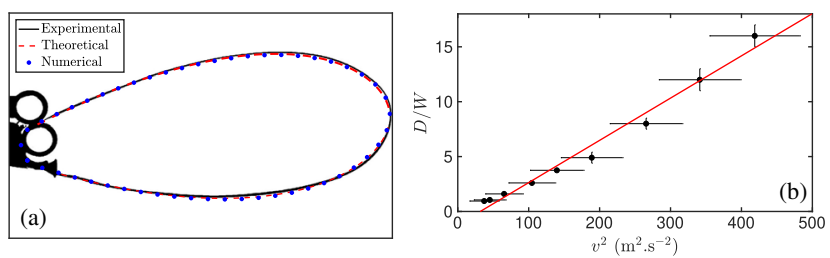

FIG. 3. (a) Comparison between the experimental, analytical, and numerical profiles, (b) values of $D / W$ for the best analytical fit to experimental profiles as a function of $v^{2}$.

$$
\begin{aligned}
& d(\tilde{T} \cos \alpha) / d \tilde{s}=D \cos \alpha, \\
& d(\tilde{T} \sin \alpha) / d \tilde{s}=W+D \sin \alpha,
\end{aligned}
$$

where $\tilde{T}=T_{\text {eff }} / T_{k}$ is the effective dimensionless tension, $\tilde{s}=s / L$ the dimensionless curvilinear coordinate, $W=g L / v^{2}$ the dimensionless weight of the string (reminiscent of a Froude number [22]) and $D=f_{\mathrm{d}} L /\left(\lambda v^{2}\right)$ the dimensionless drag. Projecting Eq. (1) on the Frenet-Serret frame indicates that $\tilde{T}$ vanishes when the string becomes vertical $(\alpha= \pm \pi / 2)$, showing that, at the turning point $O$, the tension is set by the kinetic tension $T_{k}$.

Competition between weight and drag.-First, let us focus on the regime in which the weight dominates over the drag. Figure 2(a) shows the steady-state shape of a heavy chain rotating at various velocities $\left(v=[0-15] \mathrm{m} \mathrm{s}^{-1}\right)$ : the profile is nearly independent of the velocity and very similar to the catenary obtained for a motionless chain. This result can be readily understood when neglecting drag in Eq. (1), resulting in a set of equations identical to that describing the classical hanging chain at rest, whose unique solution is the well-known catenary [7,23]. Therefore, the actual tension, $T(s)$, is given by that of a catenary, shifted by the additional uniform kinetic tension $T_{k}$, and the shape is not affected by inertial effects.

Figure 2(b) shows the steady profiles obtained with a light cotton string for various velocities (at a given propelling angle $\alpha(0)=0$ ): the shape significantly widens and rises for large velocities. In order to confirm the strong effect of aerodynamics on the shape of a propelled string, experiments were conducted in a vacuum chamber [see Fig. 2(c)]. As the pressure decreases (while the velocity and propelling angle remain unchanged), the initial nearly horizontal profile gradually turns into a hanging catenary, which again clearly demonstrates the predominant effect of air drag in the phenomenon.

Equation (1) can be numerically integrated using a simple Euler scheme and a piecewise method to avoid the singularity at the turning point $(\alpha=-\pi / 2$ and $\tilde{T}=0)$. Figure 3 compares this analytical solution to the experimental profile shown in Fig. 1, using $D / W$ as a fitting parameter $(D / W=2.4 \pm 0.05)$. The excellent agreement shows that Eq. (1) captures the physical ingredients to 


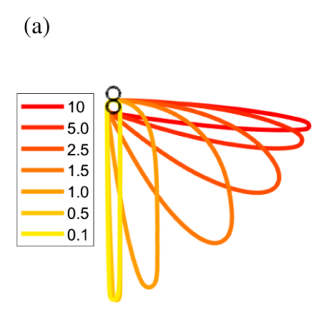

(b)

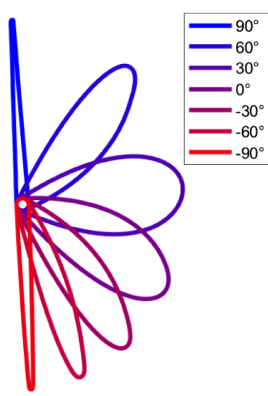

FIG. 4. (a) Evolution of the steady-state string profile as a function of $D / W$ for $\alpha(0)=0$ and (b) for various propelling angles (for $D / W=2$ ).

account for the shape of propelled strings. The best analytical fits to a series of experimental profiles with increasing propelling velocity $v$ [Fig. 3(b)] demonstrates the quadratic dependence of $D$ as a function of $v$, consistent with the typical Reynolds number Re for a $1 \mathrm{~mm}$ string moving at $v=10 \mathrm{~m} \mathrm{~s}^{-1}$ in air $(\operatorname{Re} \sim 1000)$. Therefore, the drag force per unit length may be expressed as [24] $f_{d}=\frac{1}{2} \rho C_{d} \pi R v^{2}$, where $\rho$ is the density of air, $C_{d}$ is the drag coefficient, and $R$ is the radius of the string. Figure 3(b) gives a direct measurement of $C_{d}=0.17 \pm 0.01$.

Numerical simulations. - In order to further investigate the phenomenon, time-resolved numerical simulations were performed (see Supplemental Material Video [20]). They implement a series of 1000 point masses, individually submitted to gravity and drag forces $f_{d}=\frac{1}{2} \rho C_{d} \pi R v^{2}$, and connected to their two closest neighbors by springs (stiff enough to avoid elongation of the string). The simulations are time driven and Newton's equations of motion are simultaneously integrated for each individual mass point using a Verlet algorithm. The normal forces between the masses and the wheels are computed using the same stiffness as that of the interparticle springs, and the frictional tangential forces are computed using the regularized Amontons-Coulomb law of solid friction [25]. Setting $D / W=2.4$, the steady state profile numerically obtained perfectly matches the experimental and analytical shapes, as shown in Fig. 3(a), which validates the numerical model. In this Letter, the analysis of the time-resolved numerical simulations is restricted to the steady-state profiles, allowing us to compute physical parameters, such as moments of the various forces. Nevertheless, the simulations allow the study of propagation of traveling waves or of the transient dynamic (see Supplemental Material Video part II [20]).

The steady shapes obtained numerically are shown in Fig. 4(a) for various values of $D / W$ for a horizontal propelling angle. The shape becomes increasingly horizontal as the ratio $D / W$ increases, which captures the behavior observed when the pressure or the velocity are increased. Figure 4(b) displays the shapes obtained for various propelling angles (for a given velocity) in the regime where the

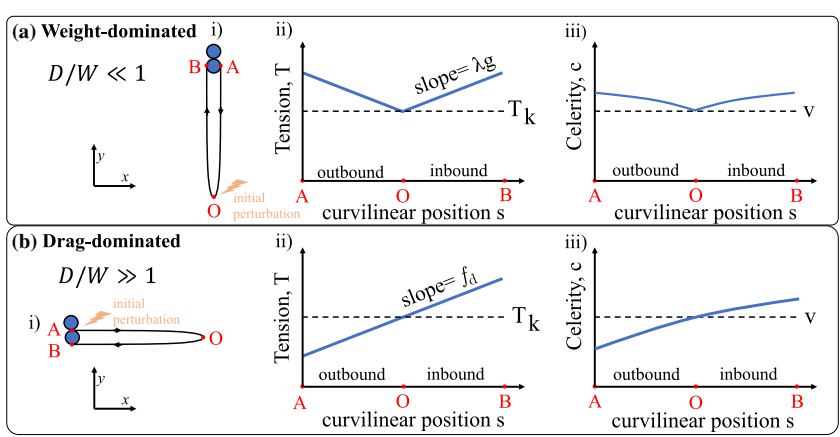

FIG. 5. Tension and wave celerity within chains and strings in (a) the weight-dominated regime and (b) the drag-dominated regime.

drag force dominates $(D / W=2)$ : the string widens when approaching a horizontal position, whereas it narrows down as it reaches a vertical position (either pointing up or down). These observations raise the question of what mechanically causes the string to rise from the vertical hanging catenary as $D / W$ increases. First, let us note that not only is there no lift acting on any segment of a string, but the sum of all drag forces along the entire string is zero (causing, however, energy dissipation, compensated by the power delivered by the motors). The explanation lies in the moment of the local drag forces. As the string rises, the moment of the weight increases (reaching its maximum as the string reaches the horizontal axis). Simultaneously, the moment of the drag force increases as the shape widens, to the point where it can balance out the moment of the weight. This is confirmed by direct computation of both moments, which shows an exact correspondence (see Supplemental Material [20] and Fig. 1). The transition between the weightdominated hanging regime and the drag-dominated regime is rather sharp and typically occurs for $1<D / W<5$ (see Supplemental Material [20] and Fig. 2).

Tension along the string.-For $D / W \ll 1$, the catenary can be approximated by two nearly vertical sections connected by a sharp turn [see Fig. 5(a)]. Equation 1(b) shows that the tension within a string moving at a velocity $v$ increases from the turning point $O$ (at which it is equal to the kinetic tension $T_{k}$ ) as the altitude $y$ increases. Therefore, along the string (i.e., with increasing curvilinear coordinate $s$ ), the tension initially decreases along the outbound branch, before increasing along the inbound branch [see Fig. 5(a-ii)]. On the other hand, for $D / W \gg 1$, the tension increases with increasing $s$, both along the outbound and inbound branches since the air drag is everywhere directed against the velocity [Fig. 5(b-ii)]. The difference in tension, due to passing through the wheels (from $B$ to $A$ ), corresponds to the mechanical work provided by the motors to compensate the energy dissipation due to the drag forces. Note, also, that the tension at point $A$ is given by $T_{k}-L f_{d} / 2$ and can mathematically become negative if the string is too long. Physically, the string would then be under compression 


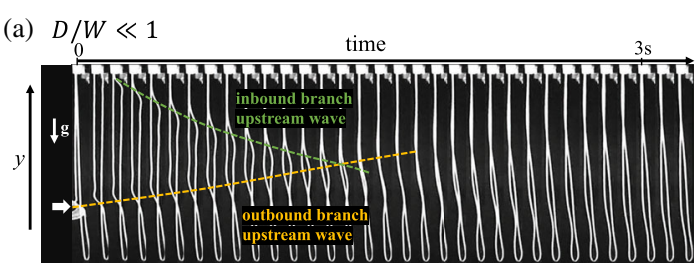

(b) $D / W \gg 1$
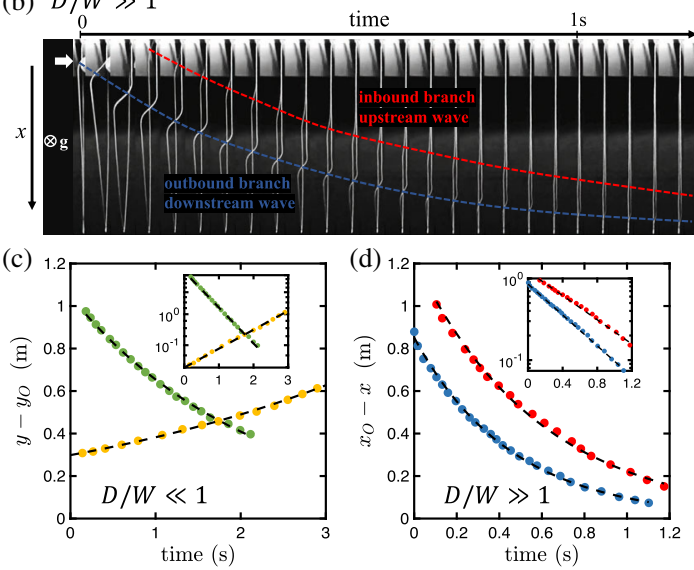

FIG. 6. Series of snapshots showing the propagation of slow waves following an initial perturbation in (a) the weightdominated regime and (b) the drag-dominated regime. (c) and (d) Time evolution of the wave front shown above.

rather than tension, causing it to buckle. This sets a minimal length-dependent velocity which ensures that the string remains under tension. This also indicates that below the critical buckling length, the profile surprisingly does not depend on the string length.

Traveling waves.-One of the most interesting features of moving chains and strings is the way traveling waves propagate in response to an initial perturbation (see Fig. 6 and Supplemental Material Video parts III and IV [20]). A local transverse perturbation on a motionless chain (or string) under tension $T(s)$ created two waves, traveling in opposite directions at a celerity $c= \pm \sqrt{T(s) / \lambda}$, to first order (see Supplemental Material II [20]). In a moving medium, the celerity of the waves combines with the speed $v$ : in the frame of reference of the lab, one wave travels at $c^{\text {fast }}=v+c$ while the other travels at $c^{\text {slow }}=v-c$. When the tension remains close to the kinetic tension, at first order, the celerity $c$ is close to the velocity $v$, see Fig. 5(a-iii) and 5(b-iii). As a consequence, the apparent velocity $c^{\text {slow }}$ is considerably smaller than $v$, corresponding to waves moving slowly in the frame of reference of the lab. In our experiments, any local perturbation will, therefore, create both a slow wave and a fast wave which quickly reflects on the propelling wheels (at point $B$ ) where it turns into an upstream slow wave along the inbound branch. This explains the existence of two slow waves, as observed in the Supplemental Material Video [20] and in Fig. 6. Note that the fast wave propagation before reflection at point $B$ explains the time lag between the two slow waves.
A series of snapshots of waves propagating on a heavy vertical beaded-chain $(D / W \ll 1)$ are shown in Fig. 6(a) rotating at $v=14.6 \mathrm{~m} \mathrm{~s}^{-1}$. An initial perturbation (near the turning point $O$ ) creates two slow waves: one traveling upward along the outbound branch (from $O$ to $A$, yellow dashed line), and one propagating downward (from $B$ to $O$, green dashed line) after reflection of the fast wave. Therefore, both slow waves travel upstream, i.e., against the velocity. Indeed, in this weight-dominated regime $(D / W \ll 1)$, $T>T_{k}$ and the apparent celerity, $c^{\text {slow }}$ is always negative [Figs. 5(a-ii) and 5(a-iii)]. A similar series of snapshots is displayed in Fig. 6(b) for a nearly horizontal light propelled string $(D / W \gg 1)$ rotating at $v=14.6 \mathrm{~m} \mathrm{~s}^{-1}$. An initial transverse perturbation (near the beginning of the outbound branch, $A$ ) also creates two slow waves both traveling towards the turning point $O$ : one wave travels downstream along the outbound branch (from $A$ to $O$, blue dashed line) whereas the wave born from the fast-wave reflection at point $B$ travels upstream along the inbound branch (from $B$ to $O$, red dashed line). In this drag-dominated regime $(D / W \ll 1)$, the tension is greater than $T_{k}$ only on the inbound branch leading to a negative apparent celerity $c^{\text {slow }}$, while $c^{\text {slow }}$ is positive on the outbound branch [Figs. 5(b-ii) and 5(b-iii)]. This transition where the celerity of waves comes to exceed that of the media in which they travel is reminiscent of the transition between subsonic and supersonic regimes for acoustic waves in a chocked Laval nozzle [26] and of the transition at unity Froude number in surface waves going through a hydraulic jump [27-29]. Note, also, that $c^{\text {slow }}$ vanishes near the turning point, where $T=T_{k}$, meaning that the waves slow down (and die out) at this point, a phenomenon clearly visible in the Supplemental Material Video [20].

In the case of a high kinetic tension (i.e., for high $v$ ), the celerity of the slow waves varies linearly with the curvilinear coordinate $s$, and the position of a wave front $s_{w f}$ is, therefore, given by (see Supplemental Material III [20])

$$
s_{w f}-s_{O}=\left(s_{0}-s_{O}\right) e^{ \pm t / \tau},
$$

where $s_{0}$ is the curvilinear position of the initial perturbation, $s_{O}$ that of the turning point $O$, and the time constant $\tau$ is given by $\tau_{W}=2 v / g$ for $D / W \ll 1$, and by $\tau_{D}=2 \lambda v / f_{d}$ for $D / W \gg 1$. The position of the wave fronts was tracked using an image-processing software [30] and the results are shown in Figs. 6(c) and 6(d). In both regimes, and along both branches, the position of the wave front is, indeed, perfectly fitted by an exponential law (dashed lines). In Fig. 6(c), one can notice that the slopes (in the semilog inset) are not identical in the outbound $\left(\tau_{W}^{o} \simeq 2.13 \mathrm{~s}\right)$ and inbound branches $\left(\tau_{W}^{i} \simeq 4.04 \mathrm{~s}\right)$, while the predicted value is $\tau_{W}=2.98 \mathrm{~s}$, which is remarkably close to the average of the two branches. This discrepancy can be attributed to the air drag, decreasing (respectively. increasing) the tension in the outbound (respectively. inbound) branch. The deviation from the predicted $\tau_{W}$ value, therefore, serves as a measure 
of $f_{d}$, which shows that, in that case, $D / W \simeq 0.3$. In the drag-dominated case, the values of $\tau_{D}$ for both branches are very close $\left(\tau_{D}^{o} \simeq 0.46 \mathrm{~s}\right.$ for the outbound branch and $\tau_{D}^{i} \simeq 0.58 \mathrm{~s}$ for the inbound branch) while the predicted value is $\tau_{D}=0.6 \pm 0.1 \mathrm{~s}$. Again, the discrepancy can be explained by the influence of the weight, which increases the tension in both branches, leading to shorter time constants than $\tau_{D}$. From this deviation, one estimates $D / W \simeq 5$.

Conclusion.-In this Letter, we have studied the effect of drag on the steady shape profiles of closed inextensible strings propelled at a constant velocity at an arbitrary angle. When the weight dominates over the drag force, the shape is close to a vertical catenary. Counterintuitively, when the drag force greatly exceeds the weight, the string impressively rises in the direction of the propelling angle. We have shown that the physical explanation lies in the balance between the moment exerted by the weight and by the drag force. Moreover, the propagation of transverse perturbations is elucidated by analyzing the variation of the tension along the string, allowing one to precisely predict the position of a wave front traveling in a string. Conversely, a study of the waves can serve as an accurate tool to infer the tension along a moving string.

The authors acknowledge support from the University of Lyon, the Université Lyon Claude Bernard, the École Normale Supérieure de Lyon. The authors are thankful to Bruce Yeany for sharing educational videos on the internet, and to H. Gayvalet, J. Sautel, J. C. Geminard, P. Wang, and A. Walbecq for fruitful discussions.

*nicolas.taberlet@ens-lyon.fr

[1] J. A. Hanna and C. D. Santangelo, Phys. Rev. Lett. 109, 134301 (2012).

[2] J. S. Biggins and M. Warner, Proc. R. Soc. A 470, 20130689 (2014).

[3] J. S. Biggins, Europhys. Lett. 106, 44001 (2014).

[4] E. G. Virga, Phys. Rev. E 89, 053201 (2014).

[5] J. Pantaleone, Am. J. Phys. 85, 414 (2017).

[6] E. G. Flekkøy, M. Moura, and K. J. Måløy, Front. Phys. 6, 84 (2018).
[7] W. Tomaszewski, P. Pieranski, and J. Geminard, Am. J. Phys. 74, 776 (2006).

[8] J. C. Geminard and L. Vanel, Am. J. Phys. 76, 541 (2008).

[9] E. Hamm and J. C. Geminard, Am. J. Phys. 78, 828 (2010).

[10] W. Tomaszewski and P. Pieranski, New J. Phys. 7, 45 (2005).

[11] J.H. Jeans, An Elementary Treatise on Theoretical Mechanics (Ginn and Company, Boston, MA, 2007).

[12] E. G. Virga, Proc. R. Soc. A 471, 20140657 (2015).

[13] W. B. Fraser, Phil. Trans. R. Soc. A 342, 439 (1993).

[14] A. Dowling, J. Fluid Mech. 187, 507 (1988).

[15] A. Dowling, J. Fluid Mech. 187, 533 (1988).

[16] P. Williams, D. Sgarioto, and P. M. Trivailo, Aerosp. Sci. Technol. 12, 347 (2008).

[17] H. Siebert, S. Gerashchenko, A. Gylfason, K. Lehmann, L. R. Collins, R. A. Shaw, and Z. Warhaft, Atmos. Res. 97, 426 (2010).

[18] J.-L. Lopes, M. Paidoussis, and C. Semler, J. Fluid Struct. 16, 715 (2002).

[19] M. Obligado and M. Bourgoin, New J. Phys. 15, 043019 (2013).

[20] See Supplemental Material at http://link.aps.org/ supplemental/10.1103/PhysRevLett.123.144501 for technical details, additional mathematical derivation and additional figures.

[21] E. de Langre, M. P. Paidoussis, O. Doare, and Y. ModarresSadeghi, J. Fluid Mech. 571, 371 (2007).

[22] J. Eggers and E. Villermaux, Rep. Prog. Phys. 71, 036601 (2008).

[23] P. Mohazzabi and J. R. Schmidt, Can. J. Phys. 77, 505 (1999).

[24] M. P. Paidoussis, Slender Structures and Axial FlowVolume 2 (Elsevier/Academic Press, London, 2003).

[25] E. Pennestri, V. Rossi, P. Salvini, and P. P. Valentini, Nonlinear Dyn. 83, 1785 (2016).

[26] R. Courant and K. Friedrichs, Supersonic Flow and Shock Waves (Springer-Verlag, New York, 1999), ISBN 978-0387-90232-6.

[27] A. D. D. Craik, R. C. Latham, M. J. Fawises, and P. W. F. Gribbon, J. Fluid Mech. 112, 347 (1981).

[28] H. G. Hornung, C. Willert, and S. Turner, J. Fluid Mech. 287, 299 (1995).

[29] J. W. M. Bush and J. M. Aristoff, J. Fluid Mech. 489, 229 (2003).

[30] Imagej, http://rsb.info.nih.gov/ij/. 